\title{
Perinatal morbidity and early neonatal mortality in twin pregnancies
}

\author{
Corinna Peter ${ }^{1}$, Paul Wenzlaff ${ }^{2}$, Jan Kruempelmann ${ }^{3}$, Gerhard Alzen $^{4}$, Eva Bueltmann ${ }^{5}$, \\ Susanne E. Gruessner ${ }^{6,7^{*}}$ \\ ${ }^{1}$ Department of Pediatric Pulmonology, Allergology and Neonatology, Hannover Medical School, Hannover, Germany \\ ${ }^{2}$ Center for Quality Assurance and Management in Health Care, Hannover, Germany \\ ${ }^{3}$ Department for Trauma and Orthopaedic Surgery, Insurance Accident and Prevention Clinic, Frankfurt am Main, Germany \\ ${ }^{4}$ Department of Pediatric Radiology, University of Giessen, Giessen, Germany \\ ${ }^{5}$ Institute of Diagnostic and Interventional Neuroradiology, Hannover Medical School, Hannover, Germany \\ ${ }^{6}$ Department of Obstetrics and Gynaecology, Justus-Liebig University of Giessen, Giessen, Germany \\ ${ }^{7}$ Department of Obstetrics, Gynaecology and Reproductive Medicine, Hannover Medical School, Hannover, Germany \\ Email: "gruessner.susanne@mh-hannover.de, ${ }^{*}$ susanne.gruessner@gmx.de
}

Received 5 October 2012; revised 7 November 2012; accepted 16 November 2012

\section{ABSTRACT}

Purpose: The purpose of this study was to investigate the impact of maternal, fetal and obstetric parameters in twin pregnancies due to chorionicity, perinatal morbidity and early neonatal mortality. Methods: Early neonatal outcome parameters were retrospectively analysed in 240 twin pregnancies $(51$ monochorionic [MC], 189 dichorionic [DC] twins) over a 7.5 years period. Beside chorionicity, we focused on risk factors affecting perinatal morbidity and early neonatal outcome in the overall study cohort and subgroups 1) late preterm and 2) pregnancies conceived by artificial fertilization (IVF/ICSI). Mixed effects logistic regression models were used for multivariate risk analyses. Results: MC vs DC pregnancies showed significantly lower birth weights $(p<0.01)$, decreased gestational ages $(p<0.01)$, increased rates of mechanical ventilation $(p<0.05)$ and higher early neonatal mortality rates $(p<0.05)$. Additional risk factors for perinatal morbidity and adverse early neonatal outcome were prematurity $(<36$ completed weeks of gestation), severe intertwin birth weight discordance > $25 \%$ and amniotic inflammation (amniotic infection syndrome [AIS]). A gestational age $>36$ completed weeks was accompanied by a decrease of early neonatal complications $(p<0.05)$. Pregnancies conceived by IVF/ ICSI didn't differ from the overall study cohort regarding the investigated risk factors. Conclusions: Twin pregnancies complicated by prematurity, AIS and severe intertwin birth weight discordance are associated with higher perinatal morbidity and adverse early neonatal outcome. In addition, MC twins are jeopardized by an increased early neonatal mortality

${ }^{*}$ Corresponding author. and therefore represent considerable challenges to both obstetricians and neonatologists. Based on our results, we recommend such twin pregnancies to be monitored and delivered at tertiary perinatal care centres to minimize perinatal morbidity and adverse early neonatal outcomes.

Keywords: Twins; Chorionicity; Obstetric Outcome; Perinatal Morbidity; Early Neonatal Outcome

\section{INTRODUCTION}

In developed nations worldwide, there has been an increase in multiple pregnancies over the last decades. Twin pregnancies are high risk pregnancies: the mortality rate in comparison to single pregnancies is 3 to 7-times higher and both short and long term morbidity rates are also increased $[1,2]$. There are several known factors categorised as maternal, fetal, or placental which affect perinatal and neonatal outcomes. Perinatal complications are mainly associated with placental arrangements. Chorionicity seems to have a significant influence on the outcome of twin pregnancies. Spontaneous abortion, stillbirth, premature birth, impaired fetal development and mortality rates are much higher in monochorionic (MC) when compared to dichorionic (DC) pregnancies [2-6].

Twin pregnancies conceived by IVF/ICSI contribute to the high caesarean section rate and higher medical costs, but they don't have major effects on adverse maternal or fetal outcomes when compared to spontaneously conceived twin pregnancies.

IVF vs spontaneously conceived twin pregnancies have an decreased proportion of MC twins but increased risk for gestational diabetes [7], delivery by caesarean 
section, preterm birth, prematurity-related respiratory complications with a longer stay and lower mean birth weight $[7,8]$. Assisted conception by IVF/ICSI is not attributable to adverse outcome but specific factors that are more common in these pregnancies, such as primiparity and fetal reduction increase the risks [9].

In addition, it has been described that birth weight discordance is an essential contributor to twin fetal death. Increasing birth weight discordance also leads to higher neonatal mortality, both in the smaller and larger twin. However, the significance of birth weight discordance on perinatal morbidity and mortality has not yet been clearly defined. Optimal delivery timing for DC twins with birth weight discordance is unreported until now. There is a lack of consensus on the precise threshold of discordance that is associated with complications.

The goal of this study was to investigate multiple maternal, fetal and obstetric prognostic parameters due to chorionicity in spontaneous and twin pregnancies conceived by IVF/ICSI with respect to perinatal morbidity and early neonatal mortality. In addition, further specific prognostic factors with impact on adverse early neonatal outcome which included both perinatal morbidity and early neonatal mortality should be selected for analysis.

\section{MATERIAL AND METHODS}

In the studied time period of 7.5 years, a total of 6698 children were delivered as a result of 6281 pregnancies at a tertiary perinatal care center. Of these pregnancies, 5889 were single births (93.8\%); there were 367 twin pregnancies $(5.8 \%)$ and 25 triplet pregnancies $(0.4 \%)$.

For the purpose of this study, all twin pregnancies with intrauterine death of one or both fetuses before 23 completed weeks of gestation and all twin pregnancies missing early antenatal sonography were excluded from the study cohort.

Nonetheless, the excluded twin pregnancies which did not have antenatal sonography were still comparable to the study population regarding maternal and fetal parameters as well as early neonatal outcomes and early neonatal mortality.

Since the exact determination of chorionicity was essential to this study and ultrasound characterization of the different placental formations is more reliable in early pregnancy, only 240 twin pregnancies were analysed in which at least one focused antenatal ultrasound (diagnosing amnionicity, chorionicity and determination of fetal gender) was performed in the first and second trimester at the tertiary perinatal care center.

Birth weight discordance was calculated using the following formula: ([birth weight larger twin] - [birth weight smaller twin])/birth weight larger twin $\times 100 \%$ [10].

As per clinical protocol, cranial ultrasound scans were performed routinely on all MC and DC twins during the first week of life (Days 1, 4, and 7). Intraventricular haemorrhage (IVH) were classified according to Papile and Burstein [11].

Data were collected retrospectively on all 240 twin pregnancies. Neonatal mortality, IVH $\geq 3^{\circ}$, infection \pm sepsis and nasal Continous Positive Airway Pressure (nCPAP)/ mechanical ventilation were evaluated as relevant perinatal morbidity and early neonatal outcome parameters within the first week of life.

In several (multivariate) analyses, thirteen independent maternal parameters were investigated (Table 1(a)). Birth weight, especially birth weight difference categorized by $<5 \%$ (no discordance), 5\% - 25\% (mild discordance) and $>25 \%$ (severe discordance) were evaluated as independent fetal parameters. Non-reassuring fetal heart rate tracings (CTG) and/or umbilical and fetal aortic Doppler results, arterial $\mathrm{pH}$-values and APGAR scores after 5 and 10 minutes were defined as independent obstetric parameters.

\section{Statistics}

For the first objective of the study - to identify determinants for the types of chorionicity (MC vs DC pregnancies) - the maternal, fetal and obstetric parameters described above were used as independent parameters by the (multivariate) procedure Genmod (Statistical Software SAS $^{\circledR}$, Version 9.3). A regression model for clustered binary responses was applied to assess the impact of $\mathrm{MC}$ versus DC together with additional maternal and twin related risk factors on mortality [12].

For the second objective of the study-to assess prognostic parameters for neonatal outcome - the four early neonatal outcome parameters defined above were selected to summarize the most severe adverse early neonatal outcomes.

Independent parameters (maternal, obstetric and fetal, see above) were used in different models for multivariate risk factor analyses by mixed effects logistic regression with $\mathrm{n}=480$ observations (=newborns) and as a random effect parameter in $\mathrm{n}=240$ twin births. A mixed effects logistic regression method was performed individually for each independent parameter of Tables 3(a) and 3(b) (with random effect parameter twin birth) to assess the odds ratios (OR) with 95\%-Confidence Intervals (95\%CI) and significancies [13]. Odds ratios regarding early neonatal outcome were adjusted for gestational age. Furthermore the two interesting subgroups of twins with gestational age $32+0-36+6$ weeks (late preterm) and twin pregnancies conceived by IVF/ICSI were analysed.

For all 480 twins, an admission to neonatal intensive care unit (NICU) or no admission as a single neonatal outcome parameter was analysed. During the different 
steps of the statistical analyses with basic and additional test procedures for (univariate) testing, the Student's t-test was used for normally distributed data, non-parametric tests with Mann-Whitney test for other distributions and Fisher's exact test for categorical ordinal data (by Statistical Package of Social Sciences SPSS ${ }^{\circledR}$, Version 19). Because of multiple testing, significancies were corrected with Bonferroni-Holm. Levels of significance were defined as $\mathrm{p}<0.05$ (= significant) and $\mathrm{p}<$ 0.01 (=high significant).

\section{RESULTS}

The independent maternal and fetal parameters of the 240 twin pregnancies and 480 newborns are listed in Tables 1(a) and (b). Out of 240 twin pregnancies, 51 (21.2\%) were MC (48 diamniotic, 3 monoamniotic) and $189(78.8 \%)$ DC pregnancies. The mean maternal age was $30.9 \pm 5.3$ years and $44.6 \%$ were primigravidas.

The mode of delivery was caesarean section in $80.4 \%$. The distribution of gender in the overall study population

Table 1. (a) Overall study cohort pregnancies and maternal parameters; (b) Overall study cohort: Obstetric and fetal parameters.

(a)

\begin{tabular}{|c|c|}
\hline \multicolumn{2}{|c|}{ Independent maternal parameters } \\
\hline Maternal parameters $(\mathrm{n}=13)$ & $\begin{array}{l}\text { Overall study cohort } \\
\text { pregnancies }(\mathrm{n}=240)\end{array}$ \\
\hline Maternal age (years) Mean \pm SD & $30.9 \pm 5.3$ \\
\hline $\begin{array}{c}<20 \text { years } \\
20-29 \text { years } \\
30-34 \text { years } \\
35-39 \text { years } \\
\geq 40 \text { years }\end{array}$ & $\begin{aligned} 9 & =3.8 \% \\
78 & =32.5 \% \\
89 & =37.1 \% \\
57 & =23.8 \% \\
7 & =2.9 \%\end{aligned}$ \\
\hline Gestational age (weeks + days) Mean $\pm \mathrm{SD}$ & $34+2 \pm 3+1$ \\
\hline Multiparity & $133=55.4 \%$ \\
\hline Hypertension & $38=15.8 \%$ \\
\hline Preeclampsia & $15=6.2 \%$ \\
\hline Tobacco use & $38=15.8 \%$ \\
\hline Tabacco use (number/day) Mean \pm SD & $0.9 \pm 2.7$ \\
\hline Artificial fertilisation (IVF/ICSI) & $146=30.4 \%$ \\
\hline PROM & $38=15.8 \%$ \\
\hline Cervical incompetence & $874=36.2 \%$ \\
\hline AIS & $15=6.2 \%$ \\
\hline Caesarean section & $193=80.4 \%$ \\
\hline Chorionicity: MC DC & $51=21.2 \% 189=78.8 \%$ \\
\hline Vaginal $\beta$-hem-streptococci & $2=0.8 \%$ \\
\hline
\end{tabular}

(b)

\begin{tabular}{|c|c|}
\hline Independent parameters & $\begin{array}{l}\text { Overall study cohort } \\
(\mathrm{n}=480)\end{array}$ \\
\hline \multicolumn{2}{|l|}{ Obstetric parameters } \\
\hline $\begin{array}{c}\text { APGAR } 5 \text { minutes } \\
\text { Mean } \pm \text { SD } \\
\text { APGAR } 5<7\end{array}$ & $\begin{array}{c}9.0 \pm 1.1 \\
13=2.7 \%\end{array}$ \\
\hline $\begin{array}{c}\text { APGAR } 10 \text { minutes } \\
\text { Mean } \pm \text { SD } \\
\text { APGAR } 10<7\end{array}$ & $\begin{array}{l}9.4 \pm 0.9 \\
4=0.8 \%\end{array}$ \\
\hline $\begin{array}{c}\text { Cord blood } \mathrm{pH} \text {-value } \\
\text { Mean } \pm \text { SD } \\
\mathrm{pH}<7.10\end{array}$ & $\begin{array}{c}7.30 \pm 0.06 \\
7=1.5 \%\end{array}$ \\
\hline Pathologic doppler and/or CTG & $119=24.8 \%$ \\
\hline \multicolumn{2}{|l|}{ Fetal parameters } \\
\hline Gender (male) & $234=48.8 \%$ \\
\hline $\begin{array}{c}\text { Gender combinations } \\
\text { (for } \mathrm{n}=240 \text { pregnancies) } \\
\mathrm{m} / \mathrm{m} \\
\mathrm{m} / \mathrm{f} \\
\mathrm{f} / \mathrm{m} \\
\mathrm{f} / \mathrm{f} \\
\text { unlike sex }\end{array}$ & $\begin{array}{l}73=30.4 \% \\
52=21.7 \% \\
36=15.0 \% \\
79=32.9 \% \\
88=36.7 \%\end{array}$ \\
\hline $\begin{array}{c}\text { Birthweight } \\
\text { Mean } \pm \text { SD } \\
\text { Birthweight 1st twin } \\
\text { Mean } \pm \text { SD } \\
\text { Birthweight 2nd twin } \\
\text { Mean } \pm \text { SD }\end{array}$ & $\begin{array}{l}2141 \pm 601 \\
2294 \pm 597 \\
1993 \pm 577\end{array}$ \\
\hline $\begin{array}{c}\begin{array}{c}\text { Birthweight } \\
\text { discordance }\end{array} \\
\text { (for } \mathrm{n}=240 \text { pregnancies) } \\
<5 \% \\
5 \%-25 \% \\
>25 \%\end{array}$ & $\begin{array}{c}66=27.5 \% \\
139=57.9 \% \\
35=14.6 \%\end{array}$ \\
\hline $\begin{array}{l}\leq 25 \% \\
>25 \%\end{array}$ & $\begin{aligned} 445 & =92.3 \% \\
35 & =7.3 \%\end{aligned}$ \\
\hline
\end{tabular}

was near evenly split with 234 male (48.8\%) and 246 female $(51.2 \%)$ newborns. The mean umbilical artery $\mathrm{pH}$ of the total cohort was $7.30 \pm 0.06$ (range $7.02-7.45$ ). The mean gestational age at birth was $34+2 \pm 3+1$ weeks and the mean birth weight for the larger twin was $2294 \pm 597 \mathrm{~g}$ (range $680-3800 \mathrm{~g}$ ) and for the smaller twin $1993 \pm 577 \mathrm{~g}$ (range $460-3160 \mathrm{~g}$ ). No intertwin birth weight discordance was seen in $132(27.5 \%)$, mild birth weight discordance in $278(57.9 \%)$ and severe birth weight discordance in $70(14.6 \%)$ of the newborns.

\subsection{Chorionicity}

In a multivariate early neonatal mortality risk factor analysis, gestational age was the most significant factor. Further logistic regression due to chorionicity and early neonatal mortality showed significance for comparison of MC and DC pregnancies $(\mathrm{p}<0.05)$ (OR 0.10 with 95\%-Confidence Interval (CI) [0.01 - 0.94] (Table 2).

Several other variables did not reach significance because of high correlation between variables which were 
Table 2. Maternal, obstetric, fetal and neonatal parameters and chorionicity.

\begin{tabular}{|c|c|c|c|}
\hline & \multicolumn{3}{|c|}{ Chorionicity } \\
\hline & DC & $\mathrm{MC}$ & p-value \\
\hline (sig.) Maternal parameters & $\mathrm{n}=189=78.9 \%$ & $\mathrm{n}=51=21.1 \%$ & \\
\hline Maternal age (years) Mean $\pm \mathrm{SD}$ & $31.3 \pm 5.2$ & $29.4 \pm 5.5$ & $\mathrm{p}<0.05^{*}$ \\
\hline $\begin{array}{c}\text { Gestational age (week + day) } \\
\text { Mean } \pm \text { SD }\end{array}$ & $34+3 \pm 3+1$ & $33+1 \pm 3+1$ & $\mathrm{p}<0.01^{*}$ \\
\hline (sig.) Obstetric parameters & $\mathrm{n}=189=78.9 \%$ & $\mathrm{n}=51=21.1 \%$ & \\
\hline Pathologic Doppler & $23=12.2 \%$ & $12=23.5 \%$ & $\mathrm{p}<0.05^{*}$ \\
\hline (sig.) Fetal parameters & $\mathrm{n}=378=78.9 \%$ & $\mathrm{n}=102=21.1 \%$ & \\
\hline $\begin{array}{l}\text { Birth weight }(\mathrm{g}) \\
\text { Mean } \pm \text { SD } \\
(\min -\max )\end{array}$ & $\begin{array}{l}2205 \pm 581 \\
(540-3250)\end{array}$ & $\begin{array}{c}1920 \pm 587 \\
(460-3800)\end{array}$ & $\mathrm{p}<0.01^{*}$ \\
\hline $\begin{array}{l}\text { Neonatal } \\
\text { parameters }\end{array}$ & $\mathrm{n}=378=78.9 \%$ & $\mathrm{n}=102=21.1 \%$ & \\
\hline Ventilation/nCPAP & $84=22.2 \%$ & $35=34.3 \%$ & $\mathrm{p}<0.05^{*}$ \\
\hline Infection \pm sepsis in first week & $20=5.3 \%$ & $10=9.8 \%$ & $\mathrm{p}=0.680$ \\
\hline $\mathrm{IVH} \geq 3^{\circ}$ & $7=1.9 \%$ & $3=2.9 \%$ & $\mathrm{p}=0.449$ \\
\hline Early neonatal mortality & \multicolumn{2}{|c|}{$\mathrm{OR}=0.10$ with $95 \%-\mathrm{CI}=0.01-0.94^{* *}$} & $\mathrm{p}=0.05^{*}$ \\
\hline
\end{tabular}

* significancies after Bonferroni-Holm correction (multiple testing); ${ }^{* *}$ adjusted for gestational age.

already in the model.

A total of five children died postnatally; one single twin in one DC pregnancy, two single twins and both twins in three MC pregnancies. The causes of death were malformation (anencephaly) in the one DC twin, and twin-to-twin transfusion syndrome (TTTS) (donor, extremely growth retarded), lung immaturity, respiratory distress or infection in the MC twins.

Comparison of $\mathrm{MC}$ and $\mathrm{DC}$ twin pregnancies revealed that women with DC twins were on average older than those with MC twins. MC twins had a significantly lower birth weight and gestational age compared to DC twins. Pathologic Doppler results were observed more often for $\mathrm{MC}$ pregnancies than for DC pregnancies. Mechanical ventilation or nCPAP was significantly more often required for $\mathrm{MC}$ vs DC twins. Incidence of infection was higher in the MC than in the DC group, but this was not significant. In total, 20 newborns delivered between $23+$ 1 to $33+4$ (mean $28+3$ weeks) were affected by different grades of intraventricular haemorrhage (IVH).

A higher grading of IVH $\geq 3^{\circ}$ was seen in 10 newborns (mean $26+3$ gestational weeks) delivered by caesarean section. The rate for IVH was not increased in the MC vs DC twins (Table 2).

TTTS developed in $5 \mathrm{MC}$ twin pregnancies. These pregnancies had to be delivered early (on average at $29+$ 1 weeks of gestation), showed a high morbidity (mechaniccal ventilation, infection) and severe birth weight discordance.

\subsection{Early Neonatal Outcome}

The present study defined adverse early neonatal outcome by the presence of early neonatal death and/or IVH $\geq 3^{\circ}$ and/or infection \pm sepsis and/or $\mathrm{nCPAP} /$ mechanical ventilation $(\mathrm{n}=125 ; 26.0 \%)$.

Using a mixed effect logistic regression model including all independent maternal, fetal and obstetric parameters a high significance was shown only for gestational age $(p<0.01)$ in a first analysis. In a second step, the same mixed effects logistic regression method was performed individually for each of the independent parameters (with random effect parameter twin birth) to assess the OR. Mean gestational age was lower for adverse early neonatal outcome than for favourable neonatal outcome. The correlated parameter birth weight showed for adverse early neonatal outcome a mean of $1612 \pm 651 \mathrm{~g}$ and for favourable neonatal outcome a mean of $2332 \pm$ early neonatal outcome $(20.0 \%)$ compared to favourable neonatal outcome $(1.1 \%)(p<0.05)$. The rate of caesarean section was higher for adverse early neonatal outcome $(96.8 \%)$ compared to favourable neonatal outcome $(74.6 \%)(\mathrm{p}<0.05)$ (Table 3(a)).

Adverse early neonatal outcome was accompanied by poor obstetric parameters. Apgar $<7$ after 5 minutes occurred more often $(8.8 \%)$ for adverse than for favourable outcome $(0.6 \%)$ ( $\mathrm{p}<0.01)$. Apgar $<7$ after 10 minutes shows corresponding rates (Table 3(b)). Cord blood analysis observed a higher rate of arterial $\mathrm{pH}$-value $<7.10$ 
Table 3. (a) Early neonatal outcome affected by maternal parameters; (b) Early neonatal outcome affected by obstetric and fetal parameters; (c) Gestational age $32+0-36+6$ weeks: Early neonatal outcome affected by maternal and fetal parameters; (d) Artificial fertilisation and early neonatal outcome; (e) Outcome = Admission to Neonatal Intensive Care Unit (NICU).

(a)

\begin{tabular}{|c|c|c|c|}
\hline \multirow[t]{2}{*}{ Independent parameters } & \multicolumn{3}{|c|}{ Early neonatal outcome } \\
\hline & Adverse outcome $^{*}(\mathrm{n}=125=26.0 \%)$ & Favourable outome $(\mathrm{n}=355=74.0 \%)$ & p-value \\
\hline \multicolumn{4}{|l|}{ Maternal parameters } \\
\hline \multirow{2}{*}{$\begin{array}{l}\text { Maternal age (years) } \\
\text { Mean } \pm \text { SD }\end{array}$} & \multicolumn{2}{|c|}{$\mathrm{OR}=1.03$ with $95 \%-\mathrm{CI}=0.96-1.11^{* * *}$} & \multirow[t]{3}{*}{$\mathrm{p}=0.411$} \\
\hline & $30.9 \pm 5.1$ & $30.8 \pm 5.1$ & \\
\hline $\begin{array}{c}<20 \text { years } \\
20-29 \text { years } \\
30-34 \text { years } \\
35-39 \text { years } \\
\geq 40 \text { years }\end{array}$ & $\begin{aligned} 4 & =3.2 \% \\
40 & =32.0 \% \\
52 & =41.6 \% \\
24 & =19.2 \% \\
5 & =4.0 \%\end{aligned}$ & $\begin{aligned} 14 & =3.9 \% \\
116 & =32.7 \% \\
126 & =35.5 \% \\
90 & =25.4 \% \\
9 & =2.5 \%\end{aligned}$ & \\
\hline Gestational age (weeks + days) & \multicolumn{2}{|c|}{$\mathrm{OR}=0.42$ with $95 \%-\mathrm{CI}=0.32-0.55$} & \multirow{2}{*}{$\mathrm{p}<0.01^{* *}$} \\
\hline Mean \pm SD & $30+6 \pm 3+3$ & $35+3 \pm 1+6$ & \\
\hline \multirow{2}{*}{ Multiparity } & \multicolumn{2}{|c|}{$\mathrm{OR}=0.95$ with $95 \%-\mathrm{CI}=0.47-2.16^{* * *}$} & \multirow{2}{*}{$\mathrm{p}=0.990$} \\
\hline & $62=49.6 \%$ & $204=57.6 \%$ & \\
\hline \multirow{2}{*}{ Artificial fertilisation (IVF/ICSI) } & \multicolumn{2}{|c|}{$\mathrm{OR}=1.06$ with $95 \%-\mathrm{CI}=0.43-2.21^{* * *}$} & \multirow{2}{*}{$\mathrm{p}=0.936$} \\
\hline & $43=34.4 \%$ & $103=29.0 \%$ & \\
\hline \multirow{2}{*}{ Hypertension } & \multicolumn{2}{|c|}{$\mathrm{OR}=0.54$ with $95 \%-\mathrm{CI}=0.17-1.68^{* * *}$} & \multirow{2}{*}{$\mathrm{p}=0.287$} \\
\hline & $12=9.6 \%$ & $64=18.0 \%$ & \\
\hline \multirow{2}{*}{ Preeclampsia } & \multicolumn{2}{|c|}{$\mathrm{OR}=0.90$ with $95 \%-\mathrm{CI}=0.19-4.2^{* * *}$} & \multirow{2}{*}{$\mathrm{p}=0.893$} \\
\hline & $7=5.6 \%$ & $23=6.5 \%$ & \\
\hline \multirow{2}{*}{ Gestational diabetes } & \multicolumn{2}{|c|}{$\mathrm{OR}=0.45$ with $95 \%-\mathrm{CI}=0.15-1.35^{* * *}$} & \multirow{2}{*}{$\mathrm{p}=0.153$} \\
\hline & $20=16.0 \%$ & $74=20.8 \%$ & \\
\hline \multirow{2}{*}{ Alcohole abuse } & \multicolumn{2}{|c|}{$\mathrm{OR}=32.8$ with $95 \%-\mathrm{CI}=0.48-2261.06^{* * *}$} & \multirow{2}{*}{$\mathrm{p}=0.106$} \\
\hline & $1=0.8 \%$ & $1=0.3 \%$ & \\
\hline Tobacco use & \multicolumn{2}{|c|}{$\mathrm{OR}=0.92$ with $95 \%-\mathrm{CI}=0.78-1.07^{* * *}$} & \multirow[t]{3}{*}{$\mathrm{p}=0.280$} \\
\hline & $0.8 \pm 2.2$ & $0.9 \pm 2.8$ & \\
\hline Tobacco use & $23=18.4 \%$ & $53=14.9 \%$ & \\
\hline \multirow{2}{*}{ Premature rupture of membranes } & \multicolumn{2}{|c|}{$\mathrm{OR}=1.24$ with $95 \%-\mathrm{CI}=0.46-3.35^{* * *}$} & $n=0662$ \\
\hline & $30=24.0 \%$ & $47=13.2 \%$ & $p-0.002$ \\
\hline Cervical incompetence & $\mathrm{OR}=1.04$ with $95 \%$ & $\mathrm{I}=0.48-2.27^{* * *}$ & $\mathrm{p}=0.920$ \\
\hline & $48=38.4 \%$ & $126=35.5 \%$ & \\
\hline ISS & $\mathrm{OR}=5.62$ with $95 \%$ - & $=1.15-30.06^{* * *}$ & $n<001^{* *}$ \\
\hline HIS & $25=20.0 \%$ & $4=1.1 \%$ & $p=0.01$ \\
\hline Caesarean section & $\mathrm{OR}=3.83$ with $95 \%$ - & $I=1.10-14.64^{* * *}$ & $\mathrm{n}<0.05^{* *}$ \\
\hline & $121=96.8 \%$ & $265=74.6 \%$ & \\
\hline & $\mathrm{OR}=1.16$ with $95 \%$ & $\mathrm{I}=0.47-2.88^{* * *}$ & \\
\hline PHonocnorionictly & $35=28.0 \%$ & $67=18.8 \%$ & $p-0.140$ \\
\hline Vaginal ß-hem-streptococci & $0=0.0 \%$ & $4=1.1 \%$ & \\
\hline
\end{tabular}

${ }^{*}$ adverse outcome $=$ early neonatal mortality and/or IVH $\geq 3^{\circ}$ and/or infection \pm sepsis and/or nCPAP/mechanical ventilation within first week; ${ }^{* *}$ significancies after Bonferroni-Holm correction (multiple testing); ${ }^{* * *}$ adjusted for gestational age. 
(b)

\begin{tabular}{|c|c|c|c|}
\hline \multirow[t]{2}{*}{ Independent parameters } & \multicolumn{3}{|c|}{ Early neonatal outcome } \\
\hline & Adverse outcome $^{*}(\mathrm{n}=125=26.0 \%)$ & Favourable outome $(\mathrm{n}=355=74.0 \%)$ & $\mathrm{p}$-value \\
\hline \multicolumn{4}{|l|}{ Obstetric parameters } \\
\hline \multirow{3}{*}{$\begin{array}{c}\text { APGAR } 5 \text { minutes } \\
\text { Mean } \pm \text { SD } \\
\text { APGAR } 5<7\end{array}$} & $8.0 \pm 1.5$ & $9.4 \pm 0.8$ & \\
\hline & \multicolumn{2}{|c|}{$\mathrm{OR}=74.15$ with $95 \%-\mathrm{CI}=4.54-1210.9^{* * *}$} & $\mathrm{p}<0.01^{* *}$ \\
\hline & $11=8.8 \%$ & $2=0.6 \%$ & \\
\hline \multirow{3}{*}{$\begin{array}{c}\text { APGAR } 10 \text { minutes } \\
\text { Mean } \pm \text { SD } \\
\text { APGAR } 10<7\end{array}$} & $8.7 \pm 1.3$ & $9.7 \pm 0.5$ & \\
\hline & \multicolumn{2}{|c|}{ no OR determinable } & $\mathrm{p}<0.01^{* *}$ \\
\hline & $4=3.2 \%$ & $0=0.0 \%$ & \\
\hline \multirow{3}{*}{$\begin{array}{c}\text { Cord blood } \mathrm{pH} \text {-value } \\
\text { Mean } \pm \text { SD } \\
\mathrm{pH}<7.10\end{array}$} & $7.3 \pm 0.08$ & $7.3 \pm 0.05$ & \\
\hline & \multicolumn{2}{|c|}{$\mathrm{OR}=28.27$ with $95 \%-\mathrm{CI}=2.70-296.17^{* * *}$} & $\mathrm{p}<0.05^{* *}$ \\
\hline & $5=4.0 \%$ & $2=0.6 \%$ & \\
\hline \multirow{2}{*}{ Pathologic doppler and/or CTG } & \multicolumn{2}{|c|}{$\mathrm{OR}=1.26$ with $95 \%-\mathrm{CI}=0.55-2.87^{* * *}$} & $\mathrm{p}<0.05^{* *}$ \\
\hline & $51=42.9 \%$ & $68=19.2 \%$ & $\mathrm{p}<0.05$ \\
\hline \multicolumn{4}{|l|}{ Fetal parameters } \\
\hline \multirow{2}{*}{ Fetus number (=1. twin) } & \multicolumn{2}{|c|}{$\mathrm{OR}=0.63$ with $95 \%-\mathrm{CI}=0.34-1.20^{* * *}$} & $n-0160$ \\
\hline & $67=53.6 \%$ & $173=48.7 \%$ & $p-0.100$ \\
\hline \multirow{2}{*}{ Gender (male) } & \multicolumn{2}{|c|}{$\mathrm{OR}=0.51$ with $95 \%-\mathrm{CI}=0.25-1.03^{* * *}$} & $\mathrm{n}=0061$ \\
\hline & $66=52.8 \%$ & $168=47.3 \%$ & $p-0.001$ \\
\hline $\begin{array}{l}\text { Gender combinations } \\
\mathrm{m} / \mathrm{m} \\
\mathrm{m} / \mathrm{f} \\
\mathrm{f} / \mathrm{m}\end{array}$ & $\begin{aligned} 43 & =34.4 \% \\
34 & =27.2 \% \\
9 & =7.2 \% \\
39 & =31.2 \%\end{aligned}$ & $\begin{aligned} 103 & =29.0 \% \\
70 & =19.7 \% \\
63 & =17.7 \% \\
119 & =33.5 \%\end{aligned}$ & \\
\hline \multirow{2}{*}{$\begin{array}{c}\mathrm{f} / \mathrm{f} \\
\text { unlike sex }\end{array}$} & \multicolumn{2}{|c|}{$\mathrm{OR}=0.77$ with $95 \%-\mathrm{CI}=0.35-1.72^{* * *}$} & $\mathrm{n}=0.532$ \\
\hline & $43=34.4 \%$ & $133=37.4 \%$ & $\mathrm{p}=0.532$ \\
\hline Birth weight $(\mathrm{g})$ Mean $\pm \mathrm{SD}$ & $1612 \pm 651$ & $2332 \pm 453$ & $\mathrm{p}<0.01^{* *}$ \\
\hline $\begin{array}{c}\text { Birth weight discordance } \\
<5 \% \\
5 \%-25 \% \\
>25 \%\end{array}$ & $\begin{array}{l}33=26.4 \% \\
57=45.6 \% \\
35=28.0 \%\end{array}$ & $\begin{aligned} 99 & =27.9 \% \\
221 & =62.3 \% \\
35 & =9.9 \%\end{aligned}$ & \\
\hline
\end{tabular}

"adverse outcome $=$ early neonatal mortality and/or IVH $\geq 3^{\circ}$ and/or infection \pm sepsis and/or nCPAP/mechanical ventilation within first week; ${ }^{* *}$ significancies after Bonferroni-Holm correction (multiple testing); ${ }^{* * *}$ adjusted for gestational age.

(c)

\begin{tabular}{|c|c|c|c|}
\hline \multicolumn{4}{|c|}{ Sub cohort: Gestational age $32+0-36+6$ weeks } \\
\hline \multirow[t]{2}{*}{ Independent parameters } & \multicolumn{3}{|c|}{ Early neonatal outcome } \\
\hline & Adverse outcome ${ }^{*}(\mathrm{n}=54=17.6 \%)$ & Favourable outcome $(\mathrm{n}=252=82.4 \%)$ & p-value \\
\hline \multicolumn{4}{|l|}{ (sig.) Maternal parameters } \\
\hline Gestational age (weeks + day) & \multicolumn{2}{|c|}{$\mathrm{OR}=0.92$ with $95 \%-\mathrm{CI}=0.81-0.97^{* * *}$} & \multirow{3}{*}{$\mathrm{p}<0.05^{* *}$} \\
\hline Mean \pm SD & $33+6 \pm 1+3$ & $34+6 \pm 1+2$ & \\
\hline Gestational age $\leq 35$ weeks & $48=88.9 \%(=22.0 \%)$ & $170=67.5 \%$ & \\
\hline \multirow{2}{*}{ AIS } & \multicolumn{2}{|c|}{$\mathrm{OR}=3.02$ with $95 \%-\mathrm{CI}=1.23-17.77^{* * *}$} & \multirow{2}{*}{$\mathrm{p}<0.05^{* *}$} \\
\hline & $7=13.0 \%$ & $3=1.2 \%$ & \\
\hline \multirow{2}{*}{ Caesarean section } & \multicolumn{2}{|c|}{$\mathrm{OR}=1.99$ with $95 \%-\mathrm{CI}=1.06-12.55^{* * *}$} & \multirow{2}{*}{$\mathrm{p}<0 ., 05^{* *}$} \\
\hline & $50=92.6 \%$ & $198=78.6 \%$ & \\
\hline \multicolumn{4}{|l|}{ (sig.) Fetal parameters } \\
\hline \multirow{3}{*}{$\begin{array}{l}\text { Gender } \\
\text { Male } \\
\text { Unlike sex }\end{array}$} & \multicolumn{2}{|c|}{$\mathrm{OR}=1.22$ with $95 \%-\mathrm{CI}=1.09-4.99^{* * *}$} & \multirow{2}{*}{$\mathrm{p}<0.05^{* *}$} \\
\hline & $34=63.0 \%$ & $109=43.3 \%$ & \\
\hline & $20=37.0 \%$ & $94=37.3 \%$ & $\mathrm{p}=1.000$ \\
\hline
\end{tabular}

${ }^{*}$ nCPAP/mechanical ventilation within first week; ${ }^{* *}$ significancies after Bonferroni-Holm correction (multiple testing); ${ }^{* * *}$ adjusted for gestational age adverse outcome $=$ early neonatal mortality and/or IVH $\geq 3^{\circ}$ and/or infection \pm sepsis and/or nCPAP/mechanical ventilation within first week. 
(d)

\begin{tabular}{|c|c|c|c|}
\hline \multicolumn{4}{|c|}{ Sub cohort: Artificial fertilisation } \\
\hline \multirow[t]{2}{*}{ Independent parameters } & \multicolumn{3}{|c|}{ Early neonatal outcome } \\
\hline & Adverse Outcome $(n=43=29.5 \%)$ & Favourable outcome $(n=103=70.5 \%)$ & p-values \\
\hline \multicolumn{4}{|l|}{ (sig.) Maternal parameters } \\
\hline Gestational age (weeks + days) & \multicolumn{2}{|c|}{$\mathrm{OR}=0.39$ with $95 \%-\mathrm{CI}=0.35-0.52^{*}$} & $\begin{array}{l}\mathrm{p}<0.001 \\
(\mathrm{p}<0.01)\end{array}$ \\
\hline Mean \pm SD & $30+3 \pm 3+5$ & $35+1 \pm 1+6$ & \\
\hline Gestational age $<28+0$ weeks & $8=18.6 \%$ & $0=0.0 \%$ & \\
\hline \multirow[t]{2}{*}{ AIS } & \multicolumn{2}{|c|}{$\begin{array}{c}\mathrm{OR}=73.00 \text { with } 95 \%-\mathrm{CI}=3.44-1548.03 \\
\mathrm{OR}=6.01 \text { with } 95 \%-\mathrm{CI}=1.22-33.65^{*}\end{array}$} & $\begin{array}{l}\mathrm{p}=0.006 \\
\mathrm{p}=0.028 \\
(\mathrm{p}<0.05)\end{array}$ \\
\hline & $11=25.6 \%$ & $3=2.9 \%$ & \\
\hline \multirow[t]{2}{*}{ Caesarean section } & \multicolumn{2}{|c|}{$\begin{array}{c}\mathrm{OR}=26.68 \text { with } 95 \%-\mathrm{CI}=0.68-1050.17 \\
\mathrm{OR}=2.77 \text { with } 95 \%-\mathrm{CI}=1.09-15.15^{*}\end{array}$} & $\begin{array}{l}\mathrm{p}=0.080 \\
\mathrm{p}=0.040 \\
(\mathrm{p}<0.01)\end{array}$ \\
\hline & $42=97.7 \%$ & $88=85.4 \%$ & \\
\hline
\end{tabular}

Adverse outcome $=$ early neonatal mortality and/or IVH3 ${ }^{\circ}$ and/or Infection Sepsis and/or NCPAP/mechanical ventilation within first week; Significancies after Bonferroni-Holm correction (multiple testing) levels of significance are defined as $\mathrm{p}<0.05$ (=significant) and $\mathrm{p}<0.01$ (=high significant); ${ }^{*}$ adjusted for gestational age.

(e)

\begin{tabular}{|c|c|c|c|}
\hline \multirow[t]{2}{*}{ Independent parameters } & \multicolumn{3}{|c|}{ Early neonatal Outcome } \\
\hline & Admission to NICU $(\mathrm{n}=260=54.2 \%)$ & No admission to NICU $(\mathrm{n}=220=45.8 \%)$ & p-value \\
\hline \multicolumn{4}{|l|}{ (sig.) Maternal parameters } \\
\hline Gestational age (weeks + days) & \multicolumn{2}{|c|}{$\mathrm{OR}=0.18$ with $95 \%-\mathrm{CI}=0.11-0.32$} & $(\mathrm{p}<0.01)$ \\
\hline Mean \pm SD & $32+4 \pm 3+2$ & $36+1 \pm 1+5$ & $\mathrm{p}<0.001$ \\
\hline \multirow{2}{*}{ Gestational age $<28+0$ weeks } & $20=7.7 \%$ & $0=0.0 \%$ & $(\mathrm{p}<0.01)$ \\
\hline & $0,8 \pm 2,2$ & $0,9 \pm 2,8$ & $\mathrm{p}=0.033$ \\
\hline \multirow[t]{2}{*}{$\begin{array}{l}\text { Tobacco use (number/day) } \\
\text { Mean } \pm \text { SD } \\
\text { Tobacco use }\end{array}$} & \multicolumn{2}{|c|}{$\begin{array}{c}\mathrm{OR}=8.17 \text { with } 95 \%-\mathrm{CI}=10.7-61.92 \\
\mathrm{OR}=2.64 \text { with } 95 \%-\mathrm{CI}=0.46-15.05(\mathrm{adj})\end{array}$} & $\begin{array}{l}\mathrm{p}=0.042 \\
\mathrm{p}=0.275\end{array}$ \\
\hline & $52=20.0 \%$ & $24=10.9 \%$ & $\begin{array}{l}\mathrm{p}=0.008 \\
(\mathrm{p}<0.01)\end{array}$ \\
\hline \multirow{2}{*}{ AIS } & \multicolumn{2}{|c|}{$\mathrm{OR}=$ not determinable } & \\
\hline & $20=11.2 \%$ & $0=0.0 \%$ & \\
\hline \multirow[t]{2}{*}{ Caesarean section } & \multicolumn{2}{|c|}{$\begin{array}{c}\mathrm{OR}=54.68 \text { with } 95 \%-\mathrm{CI}=8.26-361.96 \\
\mathrm{OR}=4.02 \text { with } 95 \%-\mathrm{CI}=0.86-18.80(\mathrm{adj})\end{array}$} & $\begin{array}{l}\mathrm{p}<0.001 \\
\mathrm{p}=0.077 \\
(\mathrm{p}<0.05)\end{array}$ \\
\hline & $235=90.4 \%$ & $151=68.6 \%$ & $\mathrm{P}<0.001$ \\
\hline \multicolumn{4}{|l|}{ (sig.) Fetal parameters } \\
\hline $\begin{array}{l}\text { Birth weight discordance } \\
<25 \%\end{array}$ & \multicolumn{2}{|c|}{$\begin{array}{c}\mathrm{OR}=11.25 \text { with } 95 \%-\mathrm{CI}=1.83-69.29 \\
\mathrm{OR}=6.12 \text { with } 95 \%-\mathrm{CI}=1.04-35.90(\mathrm{adj})\end{array}$} & $\begin{array}{l}\mathrm{p}=0.009 \\
\mathrm{p}=0.045 \\
(\mathrm{p}<0.05)\end{array}$ \\
\hline$>25 \%$ & $\begin{array}{c}231=88.8 \% \\
29=11.2 \%\end{array}$ & $\begin{aligned} 214 & =92.7 \% \\
6 & =2.7 \%\end{aligned}$ & $\begin{array}{l}\mathrm{p}<0.001 \\
(\mathrm{p}<0.05)\end{array}$ \\
\hline
\end{tabular}

$($ adj $)=$ Adjusted for gestational age; Significancies after Bonferroni-Holm correction (multiple testing).

for adverse $(4.0 \%)$ vs favourable $(0.6 \%)$ neonatal outcome $(\mathrm{p}<0.05)$. Non-reassuring CTG and/or pathological umbilical and fetal Doppler were more often diagnosed for adverse outcome (42.9\%) than for favourable outcome $(19.2 \%)(\mathrm{p}<0.05)$.
Severe birth weight discordance regarding both twins showed significant results for adverse early neonatal outcome $(\mathrm{p}<0.05)$ (Table 3(b)).

All other evaluated maternal, obstetric and fetal factors (see Tables 3(a) and (b)) had no significance when 
adverse vs favourable early neonatal outcome were compared.

After the overall analyses, the subgroup of late preterm $(32+0-36+6$ weeks of gestation) twin pregnancies was separately analysed with the same statistical approach to investigate the potential improvement of risk profile (Table 3(c)).

Significant results could be shown for mean gestational age of $33+6 \pm 1+3$ weeks for adverse neonatal outcome vs favourable outcome $(34+6 \pm 1+2$ weeks $)$.

Risk calculation of adverse early neonatal outcome showed a higher risk for 32 - 35 weeks of gestation $(22.0 \%)$ vs the group of completed 36 weeks $(6.8 \%)$. Below completed 30 weeks of gestation, the rate of adverse neonatal outcome was $\geq 72 \%$ and decreased successively with increasing gestational age and ends up with a rate of $0 \%$ above completed 38 weeks of gestation. Amniotic infection syndrome (AIS) was more often seen in the group with adverse neonatal outcome $(13.0 \%)$ than for favourable outcome $(1.2 \%)(\mathrm{p}<0.05)$ (Table 3(c)). Significantly more male fetuses $(63.0 \%)$ for adverse vs $(43.3 \%)$ for favourable neonatal outcome were observed $(\mathrm{p}<0.05)$.

Furthermore, the subgroup of IVF/ICSI pregnancies (n $=146$ observations and $\mathrm{n}=73$ twin pregnancies) was separately analysed using the same statistical approach to investigate whether they have a specific risk profile concerning adverse neonatal outcome. The mean gestational age was $30+3 \pm 3+5$ for adverse vs $35+1 \pm 1+6$ weeks $(\mathrm{p}<0.01)$. AIS was more often seen in the group with adverse neonatal outcome $(25.6 \%)$ than for favourable outcome $(2.9 \%)(\mathrm{p}<0.05)$ (Table 3(d)).

Due to chorioncity, no significant results for the subgroup of IVF/ICSI were obtained when adverse and favorable outcomes were compared.

The admission to neonatal intensive care unit (NICU) in the overall study population was analysed to examine the fetal condition postnatally; 260 newborns (54.2\%) were admitted to NICU.

Multivariate analyses showed a gestational age of $32+$ $4 \pm 3+2$ weeks for admission vs $36+1 \pm 1+5$ weeks for no admission $(\mathrm{p}<0.01)$. AIS was only recognised in the group transfer to NICU $(11.2 \%)$. Severe birth weight discordance was significant when transfer (11.1\%) and no-transfer $(2.7 \%)$ to NICU was compared $(\mathrm{p}<0.05)$ (Table 3(e)).

\section{DISCUSSION}

This single-centre study comprising 240 twin pregnancies focused on maternal, fetal and placental (MC vs DC) factors that affect perinatal morbidity and early neonatal mortality.

Our study showed a significantly higher early neonatal mortality rate for $\mathrm{MC}$ twins when compared to DC twins.
MC twins, corresponding to a decreased gestational age at delivery, had a significantly lower birth weight when compared to DC twins.

In the study, no further significant influence based on chorionicity due to adverse early neonatal outcome was identified by logistic regression analysis.

Our findings about higher early neonatal mortality rate for MC twins are in contrast to a recent report [14]. One potential explanation for the contradictionary finding might be the exclusion of pregnancies complicated by TTTS in the reported study cohort.

However, our finding are in line with various reports demonstrating less favourable pregnancy outcomes in $\mathrm{MC}$ vs DC twins and also support reports that noted a correlation between preterm delivery and higher infection and mechanical ventilation rates in MC twins. MC twins are described to be at a higher risk for fetal (even at term) and neonatal death, necrotising enterocolitis and neurological injury [15] similar to our results regarding early neonatal death. In contrast to Hack et al. but in concurrence with Lopriore et al. we did not notice a difference in the occurrence of IVH between MC and DC twins [2,16]. Irrespective of chorionicity, a third- or fourth-grade IVH was noted in 7 of 10 cases with severe intertwin discordance. The findings of IVH $\geq 3^{\circ}$ in our study could be explained by the high very early prematurity rate in these affected newborns compared to obvious advanced gestational ages and the occurence of IVH $\geq 3^{\circ}$ described by other studies [16].

Beside the factor chorionicity, our study verified that early gestational age is one of the dominant prognostic parameters for early neonatal mortality and/or perinatal morbidity.

Our study found that early neonatal outcomes, including early neonatal mortality, $\mathrm{IVH} \geq 3^{\circ}$, infection \pm sepsis and nCPAP/mechanical ventilation within the first week of life was significantly associated with early prematurity $(<32+0$ weeks $)$ but also late prematurity $(32+0-36+$ 6 weeks). Various studies describe prematurity as one of the major problems for twin pregnancy outcomes. However, reports about late preterms are mainly limited to pregnancy complication and obstetrical outcome whereas this study pinpointed an association to specific adverse neonatal outcomes. Our study results confirmed a report of moderately and late preterm twin pregnancies that encounter higher rates of neonatal morbidities compared to twins born at term [17].

Our study showed that adverse perinatal morbidity and early neonatal outcome in twin pregnancies is contributed by AIS. Overall premature rupture of membranes (PROM) complicates $7 \%$ to $8 \%$ of twin pregnancies, which led to both spontaneous and indicated preterm delivery [18]. Ananth et al. reported that despite the increase of prematurity in twin pregnancies, a concomitant 
decrease in perinatal mortality rates can be observed [19].

In our study, PROM often preceded AIS which led to significantly more indicated caesarean sections. It is plausible that the low early neonatal mortality rates we observed were a reflection of the closely antenatal surveillance combined with availability of skilled neonatal expertise in tertiary care centres.

In addition, our data support an earlier report that noted higher perinatal morbidity and early neonatal mortality rates in discordant twin pairs, even after stratification by gestational age [20]. This emphasizes the need for intensive antenatal fetal monitoring (using fetal heart rate tracings and frequently umbilical and fetal Doppler) for cases of increased risk for preterm delivery.

Our study showed that adverse obstetric outcome in combination with Apgar after 5 and 10 minutes, CTG and/or umbilical and fetal Doppler were associated with an increase of perinatal morbidity and adverse early neonatal outcomes. However, the routine obstetric parameters that were tested (APGAR values, umbilical artery $\mathrm{pH}$ values) showed that the time of delivery was chosen at an appropriate stage of gestation.

Our findings are similar to a report of Tan et al. who described that birth weight discordance of more than $25 \%$ is correlated with decreased gestational age, an increased rate of preterm birth and increased neonatal mortality in both the lighter and heavier twin [21].

Other reports described that increasing birth weight discordance leads to higher neonatal mortality in both the smaller and larger twin. Thus, significance of birth weight discordance on perinatal morbidity and mortality has not yet been clearly defined. Some reports contradict each other: for example, some investigations have challenged the significance of birth weight discordance on neonatal outcomes because they found that divergent fetal growth is associated with premature obstetric intervention. They supposed that prematurity could pose a greater threat to twins than birth weight discordance, implying that preterm twin delivery could led to a concomitant increase in neonatal morbidity $[22,23]$. They thus recommend to continue pregnancy in monochorionic diamniotic twins without clinical indication for delivery until $36-37$ weeks of gestation, avoiding complications by iatrogenic preterm birth [22].

In contrast, other investigators reported that higher neonatal mortality among twins is related to increasing levels of birth weight discordance and not entirely attributable to complications of early obstetric interventions [23].

There is a lack of consensus on the precise threshold of discordance that is associated with complications. The American College of Obstetricians and Gynecologists (ACOG) proposes the following: there is an increased risk of morbidity and death when the difference of birth weight is $15 \%-25 \%$.

With a discordance of $>25 \%$, there is an increased risk of caesarean section delivery for nonreassuring fetal heart rate tracing, neonatal acidosis, admission to NICU, and respiratory distress syndrome [14,23].

Moreover, our data support findings by Tan et al. who suggested that birth weight discordance less than $20 \%$ may be a physiologic variation, birth weight discordance ranged between $20 \%-25 \%$ may be borderline and birth weight discordance more than $25 \%$ may be a pathologic process [21]. Tobe et al. reported, that severe birth weight discordance should be added as a reference to clinical practices [23].

Our analysis of the subgroup of late preterm twin pregnancies could help to add two more risk factors for adverse early neonatal outcomes.

Firstly, the stratification of gestational weeks verified that the early neonatal complication rate was $100 \%$ below 29 pregnancy weeks. After completing 30 weeks of gestation, $2 / 3^{\text {rd }}(72 \%)$ of newborns were still troubled by postnatal complications. However, above completing 35 weeks of gestation the early neonatal complication rates dropped to $14.3 \%$ until the rate reached $0 \%$ above completed 38 weeks of gestation at delivery.

This study showed that an increase of gestational age, especially when it is more than completed 35 gestational weeks, is accompanied by a successive decrease of early neonatal complications with finally no complications after completed 38 weeks of gestation at delivery. There are only limited data for establishing optimal timing of delivery for twin pregnancies particularly due to neonatal outcomes. The Royal College of Obstetricians Gynecologists recommends delivery by 37 weeks of gestation for uncomplicated $\mathrm{MC}$ and 38 weeks of gestation for uncomplicated DC twins, whereas ACOG has not published an exact recommendation on this topic [24]. These recommendations are due to pregnancy complications followed by obstetrical complications, whereas this study focuses on neonatal outcome (see definition).

Secondly, the comparison of late preterms vs neonatal complications selected male gender as significantly correlated to adverse early neonatal outcome. This finding supports a recent study that described a male-offending factor and attributed an increased neonatal outcome risk for preterm male compared to female twins [25].

The analysis of the subgroup of IVF/ICSI pregnancies concerning a specific risk profile for adverse early neonatal complications showed no more than the above mentioned results for the overall study cohort and the late preterm subgroup. As expected, chorionicity was not a selected prognostic factor for adverse early neonatal outcome based on the fact of $97 \%$ of dichorionic pregnancies in this subgroup. 
Maternal risk factors like early gestational age, AIS, delivery mode by caesarean section and severe birth weight discordance were significantly associated with transfer of the newborns to NICU. Prematurity and respiratory morbidity accounted for the majority of perinatal morbidity and required NICU admission. Respiratory morbidity was observed despite antenatal corticosteroid exposure. Previous studies described that twin pregnancies compared to singletons are more vulnerable to respiratory morbidity because bethamethasone in twins have a shorter half-life and enhanced clearance [26].

There are both strengths and weaknesses to this study. This study benefited from large sample size, particularly by spontaneous and less by IVF/ICSI conceived DC vs MC pregnancies. In addition, the strenghts of this study lie in the multiplicity of maternal, fetal and placental risk factors and thus the likelihood so far to select more prognostic parameters that were significantly correlated to perinatal morbidity and adverse early neonatal outcome. Subgroups such as late preterm pregnancies, twin pregnancies conceived by IVF/ICSI and twin pregnancies with severe birth weight discordance provide support to precisely these risk factors for adverse early neonatal outcomes.

Our study has also some limitations that merit discussion. This study was retrospective in design and relied on first- and/or second-trimester ultrasound scan to define chorionicity. It is possible that some antenatal diagnosis by ultrasound were over-diagnosed or under-diagnosed, which has the potential to bias our results.

Although the sample of twin pregnancies was overall large, MC pregnancies and TTTS pregnancies in our cohort study group were smaller in size and that incongruity might have had influence on the results for $\mathrm{MC}$ pregnancies when compared with DC pregnancies. However, most of our demographics were similar to studies from other countries: our proportion of MC to DC twin pregnancies was $21 \%$ to $79 \%$, and not significantly different than other studies reported in the literature [27]. The percentage of children with TTTS in our study was $10 \%$ and thus slightly lower than described in the literature [28]. The relative small number of primarily MC pregnancies after IVF/ICSI could lead to an underestimation in this subgroup.

At least we determined the optimum time of delivery due to the overall study population including both DC and MC twin pregnancies without differentiation between the two types of chorionicity. However, based on the fact that the majority of the twin pregnancies in this study were DC, this limitation might therefore only cause a marginal bias to our results.

However, despite the limitations mentioned above, it might be of specific interest in clinical practice that our study showed that twins with a severe birth weight dif- ference will need admission to NICU due to an increased morbidity and mortality risk, regardless of chorionicity for both spontaneously and via IVF/ICSI conceived twin pregnancies. In concordance to another report [23], we support the appraisal that severe birth weight discordance should be included as an indication for obstetric intervention and for clinical practices.

Intensive surveillance as provided by tertiary perinatal centers offers the best preparation for optimal timing of delivery and helps to reduce morbidity and early neonatal mortality as a result of decreased gestational age [29].

\section{CONCLUSIONS}

In conclusion, it was shown that twin pregnancies conceived spontaneously or by IVF/ICSI complicated by prematurity, AIS and severe intertwin birth weight discordance are associated with higher perinatal morbidity and adverse early neonatal outcome.

Even apparently uncomplicated MC and DC twin pregnancies less than 36 weeks of gestation should be closely monitored antenatally using fetal heart rate tracings combined with umbilical and fetal Doppler velocimetry.

Cut off level of $>36$ completed weeks of gestation seemed to be the initial optimal time of delivery mainly for DC pregnancies to exclude adverse early neonatal outcomes.

Severe birth weight discordance appeared to be a key predictor for adverse neonatal outcome and should be included as an indication for obstetric intervention and for clinical practices. We recommend that such high-risk pregnancies should be referred to tertiary perinatal care centers for intense antenatal surveillance, optimal timing of delivery and thorough neonatal intensive care in an effort to reduce perinatal morbidity and adverse early neonatal outcome rates.

\section{REFERENCES}

[1] Hack, K.E., Derks, J., Elias, S., Franx, A., Roos, E., Voerman, S., Bode, C., Koopman-Esseboom, C. and Visser, G. (2008). Increased perinatal mortality and morbidity in monochorionic versus dichorionic twin pregnancies: Clinical implications of a large Dutch cohort study. British Journal of Obstetrics \& Gynaecology, 115, 58-67. doi:10.1111/j.1471-0528.2007.01556.x

[2] Hack, K.E., Koopman-Esseboom, C., Derks, J.B., Elias, S.G., de Kleine, M.J., Baerts, W., Go, A.T., Schaap, A.H., van der Hoeven, M.A., Eggink, A.J., Sollie, K.M., Weisglas-Kuperus, N. and Visser, G.H. (2009) Long-term neurodevelopmental outcome of monochorionic and matched dichorionic twins. PLoS One, 4, Article ID: e6815. doi:10.1371/journal.pone.0006815

[3] Adegbite, A., Castille, S., Ward, S. and Bajoria, R. (2004) Neuromorbidity in preterm twins in relation to chorionic- 
ity and discordant birth weight. American Journal of $\mathrm{Ob}$ stetrics and Gynecology, 190, 156-163. doi:10.1016/j.ajog.2003.07.004

[4] Glinianaia, S.V., Obeysekera, M.A., Sturgiss, S. and Bell, R. (2011) Stillbirth and neonatal mortality in monochorionic and dichorionic twins: A population-based study. Human Reproduction, 26, 2549-2557. doi:10.1093/humrep/der213

[5] Shrim, A., Weisz, B., Gindes, L. and Gagnon, R. (2010) Parameters associated with outcome in third trimester monochorionic diamniotic twin pregnancies. Journal of Obstetrics and Gynaecology Canada, 32, 429-434.

[6] Sperling, L., Kiil, C., Larsen, L., Qvist, I., Schwartz, M., Jorgensen, C., Skajaa, K., Bang, J. and Tabor, A. (2006) Naturally conceived twins with monochorionic placentation have the highest risk of fetal loss. Ultrasound in $\mathrm{Ob}$ stetrics \& Gynecology, 28, 644-652. doi:10.1002/uog. 3820

[7] Adler-Levy, Y., Lunenfeld, E. and Levy, A. (2007) Obstetric outcome of twin pregnancies conceived by in vitro fertilization and ovulation induction compared with those conceived spontaneously. European Journal of Obstetrics \& Gynecology and Reproductive Biology, 133, 173-178. doi:10.1016/j.ejogrb.2006.08.010

[8] McDonald, S.D., Han, Z., Mulla, S., Ohlsson, A., Beyene, J., Murphy, K.E. and Knowledge Synthesis Group (2010) Preterm birth and low birth weight among in vitro fertilization twins: A systematic review and meta-analyses. European Journal of Obstetrics \& Gynecology and Reproductive Biology, 148, 105-13. doi:10.1016/j.ejogrb.2009.09.019

[9] Luke, B., Brown, M.B., Alexandre, P.K., Kinoshi, T., O’Sullivan, M.J., Martin, D., Misiunas, R.B., Nugent, C., van de Ven, C., Newman, R.B., Mauldin, J.G. and Witter, F.R. (2005) The cost of twin pregnancy: Maternal and neonatal factors. American Journal of Obstetrics and Gynecology, 192, 909-915. doi:10.1016/j.ajog.2004.05.057

[10] Blickstein, I., Goldman, R.D., Smith-Levitin, M., Greenberg, M., Sherman, D. and Rydhstroem, H. (1999) The relation between inter-twin birth weight discordance and total twin birth weight. Obstetrics \& Gynecology, 93, 113116. doi:10.1016/S0029-7844(98)00343-3

[11] Papile, L., Burstein, J., Burstein, R. and Koffer, H. (1978) Incidence and evolution of subependymal and intraventricular hemorrhage. A study of infants with birth weight less than 1500 grams. The Journal of Pediatrics, 92, 529534. doi:10.1016/S0022-3476(78)80282-0

[12] Ananth, C.V., Platt, R.W. and Savitz, D.A. (2005) Regression models for clustered binary responses: Implications of ignoring the intracluster correlation in an analysis of perinatal mortality in twin gestations. Annals of Epidemiology, 15, 293-301. doi:10.1016/j.annepidem.2004.08.007

[13] Statistical Software Stata 12. http://www.stata.com/help.cgi?xtmelogit

[14] Rde, C.A.M., Brizot Mde, L., Liao, A.W., Krebs, V.L. and Zugaib, M. (2009) Early neonatal morbidity and mortality in growth-discordant twins. Acta Obstetricia et Gy- necologica Scandinavica, 88, 167-171. doi:10.1080/00016340802649808

[15] Bagchi, S. and Salihu, H.M. (2006) Birth weight discordance in multiple gestations: Occurrence and outcomes. Journal of Obstetrics and Gynaecology, 26, 291-296. doi:10.1080/01443610600594724

[16] Lopriore, E., Slaghekke, F., Vandenbussche, F.P., Middeldorp, J.M., Walther, F.J. and Oepkes, D. (2008) Cerebral injury in monochorionic twins with selective intrauterine growth restriction and/or birthweight discordance. American Journal of Obstetrics and Gynecology, 199, 628.e1-628.e5. doi:10.1016/j.ajog.2008.06.008

[17] Refuerzo, J.S., Momirova, V., Peaceman, A.M., Sciscione, A., Rouse, D.J., Caritis, S.N., Spong, C.Y., Varner, M.W., Malone, F.D., Iams, J.D., Mercer, B.M., Thorp Jr., J.M., Sorokin, Y., Carpenter, M.W., Lo, J. and Harper, M. (2010) Neonatal outcomes in twin pregnancies delivered moderately preterm, late preterm, and term. American Journal of Perinatology, 27, 537-542. doi:10.1055/s-0030-1248940

[18] Sela, H.Y. and Simpson, L.L. (2011) Preterm premature rupture of membranes complicating twin pregnancy: Management considerations. Clinical Obstetrics and Gynecology, 54, 321-329. doi:10.1097/GRF.0b013e318217d60d

[19] Ananth, C.V., Smulian, J.C., Srinivas, N., Getahun, D. And Salihu, H.M. (2005) Risk of infant mortality among twins in relation to placental abruption: contributions of preterm birth and restricted fetal growth. Twin Research and Human Genetics, 8, 524-531. doi:10.1375/twin.8.5.524

[20] Hartley, R.S., Hitti, J. and Emanuel, I. (2002) Size-discordant twin pairs have higher perinatal mortality rates than nondiscordant pairs. American Journal of Obstetrics and Gynecology, 187, 1173-1178. doi:10.1067/mob.2002.126961

[21] Tan, H., Wen, S.W., Fung, K.F.K., Walker, M. and Demissie, K. (2005) The distribution of intra-twin birth weight discordance and its association with total twin birth weight, gestational age, and neonatal mortality. European Journal of Obstetrics \& Gynecology and Reproductive Biology, 121, 27-33. doi:10.1016/j.ejogrb.2004.10.012

[22] Smith, N.A., Wilkins-Haug, L., Santolaya-Forgas, J., Acker, D., Economy, K.E., Benson, C.B. and Robinson, J.N. (2010) Contemporary management of monochorionic diamniotic twins: Outcomes and delivery recommendations revisited. American Journal of Obstetrics and Gynecology, 203, 133.e1-133.e6. doi:10.1016/j.ajog.2010.02.066

[23] Tobe, R.G., Mori, R., Shinozuka, N., Kubo, T. and Itabashi, K. (2010) Birthweight discordance, risk factors and its impact on perinatal mortality among Japanese twins: Data from a national project during 2001-2005. Twin Research and Human Genetics, 13, 490-494. doi:10.1375/twin.13.5.490

[24] Royal College of Obstetricians Gynaecologists (2008) Management of monochorionic twins.

http://www.aogm.org.mo/assets/Uploads/aogm/Guideline 
s/RCOG-UK/T51-Management-Monochorionic-Twin-Pr egnancy2008a.pdf

[25] Melamed, N., Yogev, Y. and Glezerman, M. (2009) Effect of fetal sex on pregnancy outcome in twin pregnancies. Obstetrics \& Gynecology, 114, 1085-1092. doi:10.1097/AOG.0b013e3181bd8874

[26] Choi, S.J., Song, S.E., Seo, E.S., Oh, S.Y., Kim, J.H. and Roh, C.R. (2009) The effect of single or multiple courses of antenatal corticosteroid therapy on neonatal respiratory distress syndrome in singleton versus twin pregnancies. The Australian and New Zealand Journal of Obstetrics and Gynaecology, 49, 173-179. doi:10.1111/j.1479-828X.2009.00970.x

[27] Baghdadi, S., Gee, H., Whittle, M.J. and Khan, K.S.
(2003) Twin pregnancy outcome and chorionicity. Acta Obstetricia et Gynecologica Scandinavica, 82, 18-21. doi:10.1034/j.1600-0412.2003.820103.x

[28] Jain, V. and Fisk, N.M. (2004) The twin-twin transfusion syndrome. Clinical Obstetrics and Gynecology, 47, 181202. doi:10.1097/00003081-200403000-00020

[29] Lewi, L., Gucciardo, L., Huber, A., Jani, J., Van Mieghem, T., Doné, E., Cannie, M., Gratacós, E., Diemert, A., Hecher, K., Lewi, P. and Deprest, J. (2008) Clinical outcome and placental characteristics of monochorionic diamniotic twin pairs with early- and late-onset discordant growth. American Journal of Obstetrics and Gynecology, 199, 511.e1-511.e7. doi:10.1016/j.ajog.2008.04.022 